\title{
VOLTERRA INTEGRAL EQUATIONS WITH HIGHLY OSCILLATORY KERNELS: A NEW NUMERICAL METHOD WITH APPLICATIONS*
}

\author{
LUISA FERMO ${ }^{\dagger}$ AND CORNELIS VAN DER MEE ${ }^{\dagger}$
}

\begin{abstract}
The aim of this paper is to present a Nyström-type method for the numerical approximation of the solution of Volterra integral equations of the second kind having highly oscillatory kernels. The method is based on a mixed quadrature scheme which combines the classical product rule with a dilation quadrature formula. The convergence and the stability of the method are investigated and the accuracy of the presented approach is assessed by some numerical tests. The proposed procedure is also applied to the computation of initial scattering data related to the initial value problem associated to the Korteweg-de Vries equation.
\end{abstract}

Key words. Volterra integral equation, highly oscillatory kernels, Nyström method, mixed quadrature scheme, Korteweg-de Vries equation

AMS subject classifications. 65R20, 41A05, 45D05.

1. Introduction. Let us consider the following Volterra integral equation

$$
f(s)+\int_{a}^{s} k(\omega(t-s)) f(t) d t=g(s), \quad s \in[a, b], \quad 0 \neq \omega \in \mathbb{R},
$$

where $f$ is the unknown function, $g$ is a given right-hand side defined on $[a, b]$, and $k$ is a known highly oscillatory kernel defined on $\Delta=\{(t, s): a \leq t \leq s \leq b\}$ depending on a real positive fixed parameter $\omega$. Examples of such kernels are

$$
k(\omega(t-s))=\frac{\sin (\omega(t-s))}{\omega} \quad \text { and } \quad k(\omega(t-s))=J_{\nu}(\omega(t-s)),
$$

$J_{\nu}$ being the Bessel function of the first kind of order $\nu \geq 0$.

Integral equations of the type (1.1) arise in several engineering problems including wave propagation, quantum mechanics, acoustic scattering, signal processing, and image recognition $[8,18]$. A specific example is given by the following differential problem associated to the Schrödinger equation on the line

$$
\left\{\begin{array}{l}
-u^{\prime \prime}(\omega ; x)+q(x) u(\omega ; x)=\omega^{2} u(\omega ; x), \quad x \in \mathbb{R}, \\
u(\omega ; 0)=\alpha \\
u^{\prime}(\omega ; 0)=\beta
\end{array}\right.
$$

whose solution $u(\omega ; x)$ satisfies the following equation

$$
u(\omega ; y)-\int_{0}^{y} \frac{\sin (\omega(y-x))}{\omega} q(x) u(\omega ; x) d x=\alpha \cos (\omega y)+\beta \frac{\sin (\omega y)}{\omega} .
$$

*Received July 29, 2020. Accepted March 16, 2021. Published online on April 23, 2021. Recommended by Sotirios Notaris. The authors are partially supported by Regione Autonoma della Sardegna research project "Algorithms and Models for Imaging Science [AMIS]" (RASSR57257, intervento finanziato con risorse FSC 20142020 - Patto per lo Sviluppo della Regione Sardegna). Luisa Fermo is member of the INdAM Research group GNCS and is also partially supported by Fondazione di Sardegna 2017 research project "Algorithms for Approximation with Applications [Acube]" and INdAM-GNCS 2020 project "Approssimazione multivariata ed equazioni funzionali per la modellistica numerica". Cornelis van der Mee is member of the INdAM Research group GNFM and is partially supported by Fondazione di Sardegna 2017 research project "Integro-differential Equations and Non-Local Problems". This research has been accomplished within the RITA "Research ITalian network on Approximation".

$\dagger$ Department of Mathematics and Computer Science, University of Cagliari, Via Ospedale 72, 09124, Italy, (fermo@unica.it, cornelis@krein.unica.it). 
A further specific application (see Section 6) concerns the direct scattering problem for the initial value problem associated to the Korteweg-de Vries (KdV) equation [19]

$$
\left\{\begin{array}{l}
q_{t}-6 q q_{x}+q_{x x x}=0, \quad x \in \mathbb{R}, \quad t>0, \\
q(x, 0)=q_{0}(x),
\end{array}\right.
$$

where $q(x, t)$ is the potential to determine at the time $t$, and the subscripts $x$ and $t$ designate partial derivatives with respect to position and time. In fact, equations of type (1.1) come into play to determine some initial scattering data like the transmission and reflection coefficients.

The numerical treatment of equation (1.1) is quite delicate especially when $\omega \gg 1$, i.e., when the kernel function is highly oscillatory. In this case, the computation of the integral by traditional quadrature formulae is exceedingly difficult and becomes expensive: for large values of $\omega$, the integrand oscillates rapidly and the number of evaluations grows linearly with $\omega$; see, for instance, $[9,16,20]$. Consequently, numerical methods based on the classical quadrature formulae are not practicable for this kind of equation.

Nowadays, many methods have been proposed for computing highly oscillatory integrals so that the accuracy of the computation increases as the oscillation parameter $\omega$ tends to infinity: asymptotic methods [17], Filon-type methods [12, 13, 22, 26], Levin-type methods [21, 25], numerical steepest descent [15], and dilation techniques [9]. With the help of these numerical approaches, several methods were developed to approximate the solution of highly oscillatory Volterra integral equations; see [6, 31, 32]. In [6], the authors analyze the oscillatory properties of the solution of Volterra integral equations having specific oscillatory kernels. In [31, 32], several numerical methods, based on Filon, piecewise constant, and linear collocation techniques, are presented to approximate the solution of Volterra integral equations with highly oscillatory Bessel kernels.

In this paper, we propose a Nyström-type method based on a mixed quadrature formula. Following [5], we construct the method by using a product quadrature formula based on the zeros of Legendre polynomials. In order to compute the coefficients of such a rule we use a "dilation" quadrature formula; see [9]. It consists of a preliminary dilation of the domain and a subsequent repartition of the integration interval. For such a rule, we provide a new error estimate thanks to new estimates of the derivatives of the fundamental Lagrange polynomials which, to our knowledge, has never been proved before. The proposed Nyström-type method, whose convergence and stability are proved in spaces of continuous functions, leads to a wellconditioned linear system whose order does not depend on the magnitude of the parameter $\omega$, and the error is of the order of the best polynomial approximation of the unknown function, independently of the value of $\omega$. We apply the proposed procedure to compute some initial scattering data related to problem (1.2).

We remark that in [22] an interesting quadrature formula has been introduced to compute highly oscillatory integrals whose integrand function may have weak singularities. The main idea is to divide the interval of integration into subintervals, according to the parameter $\omega$ and the singularity of the integrand. If such a quadrature scheme is adopted in a Nyström type method for solving Volterra integral equations (1.1), competitive results are obtained in the case where the known functions are smooth. Moreover, the quadrature rule given in [22] allows one to treat singularities. Therefore, it would be of great interest to apply such a rule to approximate the solution of Volterra integral equations having kernels with singularities. It will be a topic of future research.

This paper is organized in seven sections. In Section 2, we define the function spaces in which we look for the solution of equation (1.1), we recall some basic facts of Legendre polynomials and Gauss-Legendre quadrature formulas, and we prove new estimates for the derivatives of the involved polynomials. In Section 3, we focus on the dilated product 
quadrature formula, and in Section 4 we apply such a rule to the method we propose to solve equation (1.1). We conclude our paper with several numerical tests (Sections 5 and 6) and with the proofs of our main results in Section 7.

\section{Preliminaries.}

2.1. Function spaces. Let us denote by $C^{0}([a, b])$ the space of all continuous functions in the interval $[a, b]$ equipped with the norm

$$
\|f\|_{\infty}=\sup _{x \in[a, b]}|f(x)|,
$$

and by $C^{r}([a, b]), r \in \mathbb{N}$, the space of functions having a continuous $r$-th derivative in $[a, b]$.

Let us also introduce the error of best polynomial approximation of a given function $f \in C^{0}([a, b])$, defined as

$$
E_{m}(f)_{\infty}=\inf _{P_{m} \in \mathbb{P}_{m}}\left\|f-P_{m}\right\|_{\infty}
$$

where $\mathbb{P}_{m}$ denotes the space of algebraic polynomials of degree at most $m$. It is well-known that, according to the Weierstrass theorem [23, p. 3],

$$
\lim _{m \rightarrow \infty} E_{m}(f)=0, \quad \forall f \in C^{0}([a, b]),
$$

and (see, for instance, [30, p. 261, formula (22)] and [23, p. 172, formula (2.5.22)])

$$
E_{m}(f) \leq \frac{\mathcal{C}}{m^{r}}\left\|f^{(r)}\right\|_{\infty}, \quad \forall f \in C^{r}([a, b]),
$$

where the constant $\mathcal{C}$ only depends on $r$.

2.2. Legendre polynomials. Let $\left\{P_{m}\right\}_{m \in \mathbb{N}}$ be the sequence of orthogonal Legendre polynomials on $(-1,1)$ obeying the following three-term recurrence relation

$$
\left\{\begin{array}{l}
P_{-1}(x)=0, P_{0}(x)=1, \\
(m+1) P_{m+1}(x)=(2 m+1) x P_{m}(x)-m P_{m-1}(x), \quad m=0,1,2, \ldots
\end{array}\right.
$$

It is well-known that, for this sequence,

$$
\int_{-1}^{1} P_{m}(x) P_{n}(x) d x=\frac{2}{2 m+1} \delta_{m n}
$$

where $\delta_{m n}$ is the Kronecker delta, which takes the value 0 if $m \neq n$ and the value 1 if $m=n$.

By differentiating (2.1) with respect to $x$ and taking into account that

$$
x P_{m}^{\prime}(x)-P_{m-1}^{\prime}(x)=m P_{m}(x),
$$

we obtain

$$
P_{m+1}^{\prime}(x)=(2 m+1) P_{m}(x)+P_{m-1}^{\prime}(x) .
$$

It is also well-known that if we rewrite (2.1) as

$$
x P_{m}(x)=\frac{m}{2 m+1} P_{m-1}(x)+\frac{m+1}{2 m+1} P_{m+1}(x), \quad m=0,1,2, \ldots,
$$


and we consider the first $m=0,1, \ldots, n-1$ equations, then (2.4) can be interpreted as the following system of linear equations

$$
\left(x \mathbb{I}_{n}-\mathbb{T}_{n}\right) \mathbf{P}_{n}=\frac{n}{2 n-1} P_{n}(x) \mathbf{e}_{n},
$$

where $\mathbf{P}_{n}(x)=\left[P_{0}(x), P_{1}(x), \ldots, P_{n-1}(x)\right]^{T}, \mathbf{e}_{n}=[0,0, \ldots, 0,1]^{T}, \mathbb{I}_{n}$ is the identity matrix of order $n$, and $\mathbb{T}_{n}$ is the $n \times n$ tridiagonal matrix whose entries are defined as

$$
\left[\mathbb{T}_{n}\right]_{i, j=1}^{n}= \begin{cases}\beta_{i}=\frac{i+1}{2 i+1}, & i=j-1, \\ 0, & i=j, \\ \alpha_{i}=\frac{i}{2 i+1}, & i=j+1\end{cases}
$$

and whose eigenvalues are the zeros of $P_{n}(x)$.

Let us also recall the values of the Legendre polynomials and their first derivative at \pm 1

$$
P_{m}(1)=1, \quad P_{m}^{\prime}(1)=\frac{m(m+1)}{2}, \quad P_{m}(-1)= \begin{cases}1, & n=2 m, \\ -1, & n=2 m+1 .\end{cases}
$$

If we look at the derivatives of $P_{m}$, by the well-known Markov inequality (see, for instance, [14] and [28, Problem 5]), we can assert that

$$
\left|P_{m}^{(j)}(x)\right| \leq m^{2 j}
$$

since $\left\|P_{m}\right\|_{\infty}=1$.

In the next proposition we prove the above estimate by giving an alternative proof that, to our knowledge, has never been proposed before. The proof is by induction; its starting point is that, by (2.3), we can write

$$
P_{m}^{\prime}(x)=\sum_{r=0}^{p} c_{m r} P_{m-2 r-1}(x), \quad \text { with } \quad p= \begin{cases}\frac{1}{2}(m-1), & m \text { is odd, } \\ \frac{1}{2} m-1, & m \text { is even, }\end{cases}
$$

where, by (2.2) and (2.7), the coefficients $c_{m r}$ are given by

$$
c_{m r}=\frac{2 m-4 r-1}{2} \int_{-1}^{1} P_{m}^{\prime}(x) P_{m-2 r-1}(x) d x=2 m-4 r-1 .
$$

Moreover, by (2.7), we also deduce that

$$
\sum_{r=0}^{p} c_{m r}=\frac{m(m+1)}{2}
$$

PRoposition 2.1. Let $P_{m}$ be the orthogonal Legendre polynomial of degree $m$. Then, for each $j \geq 0$ we have

$$
\left\|P_{m}^{(j)}\right\|_{\infty} \leq m^{2 j} .
$$


2.3. The Lagrange interpolation process associated to Legendre polynomials. Let us consider the sequence of orthonormal Legendre polynomials

$$
p_{m}(x)=\frac{P_{m}(x)}{\left\|P_{m}\right\|}=\gamma_{m} x^{m}+\text { lower degree terms },
$$

where $\gamma_{m}=\sqrt{m+\frac{1}{2}} \frac{1}{2^{m}}\left(\begin{array}{c}2 m \\ m\end{array}\right)$ and $\left\|P_{m}\right\|=\sqrt{\frac{2}{2 m+1}}$; see (2.2).

Let us denote by $-1<x_{1}<x_{2}<\cdots<x_{m}<1$ the zeros of $p_{m}$ and let us introduce the Lagrange polynomial based on these nodes, namely

$$
\mathcal{L}_{m}(f, x)=\sum_{k=1}^{m} \ell_{k}(x) f\left(x_{k}\right),
$$

where $\ell_{k}$ is the $k$-th fundamental Lagrange polynomial defined as

$$
\ell_{k}(x)=\prod_{\substack{i=1 \\ i \neq k}}^{m} \frac{\left(x-x_{i}\right)}{\left(x_{k}-x_{i}\right)}=\frac{p_{m}(x)}{p_{m}^{\prime}\left(x_{k}\right)\left(x-x_{k}\right)} .
$$

The next proposition states an estimate for the derivatives of $\ell_{k}$.

PROPOSITION 2.2. Let $\ell_{k}$ be the $k$-th fundamental Lagrange polynomial associated to the Legendre nodes defined in (2.12). Then, for each $x \in[-1,1]$ we have

$$
\left|\ell_{k}^{(r)}(x)\right| \leq \sqrt{\frac{\pi}{2}} m^{2 r+\frac{1}{2}}\left(1-x_{k}^{2}\right)^{\frac{1}{4}}
$$

Let us underline that the modulus of the $r$-th derivative $\ell_{k}^{(r)}$ may also be estimated by using Markov's inequality, but doing so we would obtain

$$
\left|\ell_{k}^{(r)}(x)\right| \leq m^{2 r}\left\|\ell_{k}\right\|_{\infty}
$$

2.4. The Gauss-Legendre formula. Let us consider the classical Gauss-Legendre formula

$$
\int_{a}^{b} f(t) d t=\sum_{i=1}^{n} \lambda_{i} f\left(t_{i}\right)+\mathcal{R}_{n}(f),
$$

where the $t_{i}$ are the zeros of the Legendre polynomial in $[a, b]$, i.e.,

$$
t_{i}=a+\frac{(b-a)}{2}\left(x_{i}+1\right), \quad x_{i} \in(-1,1),
$$

the Christoffel numbers $\lambda_{i}$ in $[a, b]$ are given by

$$
\lambda_{i}=\frac{b-a}{2} \int_{-1}^{1} \ell_{i}(t) d t
$$

and $\mathcal{R}_{n}$ stands for the remainder term. The following theorem holds.

THEOREM 2.3. [23, Theorem 5.1.5] Let $f \in C^{2 n}([a, b])$. There exists $\xi \in(a, b)$ such that the following formula holds for the remainder term

$$
\mathcal{R}_{n}(f)=\frac{f^{(2 n)}(\xi)}{(2 n) !} \int_{a}^{b} \prod_{j=1}^{n}\left(t-t_{j}\right)^{2} d t
$$


where $t_{j}$ are the zeros of the $n$-th order orthogonal polynomial with respect to the Lebesgue measure on $(a, b)$.

Hence, according to the above result, being

$$
P_{n}(x)=\frac{1}{2^{n}}\left(\begin{array}{c}
2 n \\
n
\end{array}\right) \prod_{j=1}^{n}\left(x-x_{j}\right),
$$

taking into account (2.2) and the well-known Stirling formula

$$
n ! \sim \sqrt{2 \pi n}\left(\frac{n}{e}\right)^{n}
$$

we can state that

$$
\begin{aligned}
\left|\mathcal{R}_{n}(f)\right| & \leq \frac{\left\|f^{(2 n)}\right\|_{\infty}}{(2 n) !} \int_{a}^{b} \prod_{j=1}^{n}\left(t-t_{j}\right) d t \\
& =\frac{\left\|f^{(2 n)}\right\|_{\infty}}{(2 n) !}\left(\frac{b-a}{2}\right)^{2 n+1}\left(\frac{2^{n}(n !)^{2}}{(2 n) !}\right)^{2} \int_{-1}^{1} P_{n}^{2}(x) d x \\
& =\left\|f^{(2 n)}\right\|_{\infty}\left(\frac{b-a}{2}\right)^{2 n+1} \frac{2^{2 n+1}(n !)^{4}}{[(2 n) !]^{2}(2 n+1) !} \\
& \simeq\left\|f^{(2 n)}\right\|_{\infty}\left(\frac{b-a}{2}\right)^{2 n+1} \frac{\pi}{2^{2 n}(2 n) !} .
\end{aligned}
$$

3. A dilated product quadrature rule. In this section we introduce a quadrature rule for the approximation of the integral

$$
(\mathcal{V} f)(s)=\int_{a}^{s} k(\omega(t-s)) f(t) d t
$$

The idea is to apply a product quadrature rule whose coefficients are approximated by using a suitable quadrature scheme [9, 27]. Such "mixed" formulas are not new and have already been applied to integrals having "nearly singular kernels", which arise in specific Fredholm integral equations $[11,24]$.

Let us approximate the unknown function $f$ by using the Lagrange polynomial (2.11) associated with the Legendre nodes $\left\{t_{j} \in(a, s)\right\}_{j=1}^{m}$. In this way we obtain the following $m$-point product rule [23, p. 347]

$$
\left(\mathcal{V}_{m} f\right)(s)=\sum_{j=1}^{m} c_{j}(s) f\left(t_{j}\right),
$$

where the coefficients $c_{j}(s)$ are defined by

$$
c_{j}(s)=\int_{a}^{s} \ell_{j}(t) k(\omega(t-s)) d t, \quad j=1,2, \ldots, m .
$$

3.1. The approximation of the coefficients. Let us now focus on the approximation of the coefficients $c_{j}$ introduced in (3.3), which is necessary whenever they cannot be evaluated analytically. The following example shows the difficulty we have in approximating them by using the classical $n$-point Gauss-Legendre formulae (2.14). 
EXAMPLE 3.1. Let us consider the coefficients (3.3) in the case $[a, b]=[-1,1]$, and let the kernel $k$ coincide with the function $\frac{\sin (\omega(t-s))}{\omega}$. Setting

$$
h_{j}(t, s)=\ell_{j}(t) \frac{\sin (\omega(t-s))}{\omega},
$$

according to (2.14) we can write

$$
c_{j}(s)=\int_{-1}^{s} h_{j}(t, s) d t=\sum_{\nu=1}^{n} \lambda_{\nu} h_{j}\left(t_{\nu}, s\right)+\mathcal{R}_{n}\left(h_{j}, s\right)=: c_{j n}(s)+\mathcal{R}_{n}\left(h_{j}, s\right),
$$

where $t_{\nu}$ and $\lambda_{\nu}$ are defined on the integration interval $(-1, s)$. Since the integrand $h$ is an analytic function, by virtue of (2.16) we have

$$
\left|\mathcal{R}_{n}\left(h_{j}, s\right)\right| \leq\left\|h_{j}^{(2 n)}\right\|_{\infty}\left(\frac{s+1}{2}\right)^{2 n+1} \frac{\pi}{2^{2 n}(2 n) !} .
$$

Therefore, by applying the generalized Leibniz rule and taking into account estimate (2.13), we obtain

$$
\begin{aligned}
\left|\frac{\partial^{2 n}}{\partial t^{2 n}} h_{j}(t, s)\right| & \leq \sum_{i=0}^{2 n}\left(\begin{array}{c}
2 n \\
i
\end{array}\right)\left|\ell_{j}^{(2 n-i)}(t)\right| \cdot\left|\frac{\partial^{i}}{\partial t^{i}} \frac{\sin (\omega(t-s))}{\omega}\right| \\
& \leq \sqrt{\frac{m \pi}{2}}\left(1-x_{j}^{2}\right)^{\frac{1}{4}} \sum_{i=0}^{2 n}\left(\begin{array}{c}
2 n \\
i
\end{array}\right) m^{2(2 n-i)} \omega^{i-1} \\
& =\sqrt{\frac{\pi}{2}}\left(1-x_{j}^{2}\right)^{\frac{1}{4}} \omega^{2 n-\frac{1}{2}} \sqrt{\frac{m}{\omega}}\left(\frac{m^{2}}{\omega}+1\right)^{2 n} \\
& \leq \sqrt{\pi}(2 \omega)^{2 n-\frac{1}{2}}
\end{aligned}
$$

where $m \ll \omega$. Consequently, by (3.5) and (2.15), we have

$$
\left|\mathcal{R}_{n}\left(h_{j}, s\right)\right| \leq \frac{\pi}{2} \frac{(s+1)}{2}\left(\frac{(s+1) \omega e}{4 n}\right)^{2 n} \sqrt{\frac{1}{2 n \omega}} .
$$

From this estimate, we deduce that to obtain accurate results, the Gauss-Legendre rule must be based on a number of quadrature points $n>\omega$. By way of example, we display in Table 3.1 the numerical results we obtain in the case $\omega=3000, s \equiv 1$, and $m=256$.

TABLE 3.1

The approximation $c_{j n}(1)$ of coefficients (3.4), for $j=1,5,10$.

\begin{tabular}{c|ccc}
$n$ & $c_{1 n}(1)$ & $c_{5 n}(1)$ & $c_{10 n}(1)$ \\
\hline 4 & $2.84776143374 \mathrm{e}-07$ & $1.90788056269 \mathrm{e}-05$ & $1.22828128312 \mathrm{e}-05$ \\
8 & $1.18955555474 \mathrm{e}-07$ & $-8.30256352523 \mathrm{e}-07$ & $-1.72235386568 \mathrm{e}-05$ \\
16 & $9.67712555110 \mathrm{e}-07$ & $8.62577021319 \mathrm{e}-06$ & $-2.87307048627 \mathrm{e}-06$ \\
32 & $1.63600016700 \mathrm{e}-06$ & $-6.34956805249 \mathrm{e}-06$ & $-1.23312398060 \mathrm{e}-05$
\end{tabular}

The previous example shows that if we approximate the coefficients by a Gauss-Legendre formula, as theoretically expected, there is no convergence for small values of $n$ and, in some cases, there is a change of sign in the computed result. 
Then, to overcome this problem, we use a dilated quadrature rule introduced for the first time in [9]. The technique consists of dilating the domain of integration by making the change of the variable $t=\frac{z}{\omega}$. In this way, we can write

$$
c_{j}(s)=\frac{1}{\omega} \int_{\omega a}^{\omega s} \ell_{j}\left(\frac{z}{\omega}\right) k(z-\omega s) d z,
$$

from which, by partitioning the integral into $N$ subintervals of a certain length $\delta$, we have

$$
c_{j}(s)=\frac{1}{\omega} \sum_{\iota=1}^{N} \int_{\omega a+(\iota-1) \delta}^{\omega a+\iota \delta} \ell_{j}\left(\frac{z}{\omega}\right) k(t-\omega s) d z .
$$

Then, if we remap each integral into $[a, s]$ by using the map

$$
\sigma_{\iota}:[\omega a+(\iota-1) \delta, \omega a+\iota \delta] \rightarrow[a, s],
$$

defined by

$$
t=\sigma_{\iota}(z)=\frac{s-a}{\delta}(z-\omega a)+s-(s-a) \iota,
$$

we reach the expression

$$
c_{j}(s)=\frac{\delta}{(s-a) \omega} \sum_{\iota=1}^{N} \int_{a}^{s} \ell_{j}\left(\frac{\sigma_{\iota}^{-1}(t)}{\omega}\right) k\left(\sigma_{\iota}^{-1}(t)-\omega s\right) d t .
$$

At this point we approximate each integral by using the $n$-point Gauss-Legendre formula (2.14), to obtain

$$
c_{j}^{n}(s)=\frac{\delta}{(s-a) \omega} \sum_{\iota=1}^{N} \sum_{j=1}^{n} \lambda_{j} \ell_{j}\left(\frac{\sigma_{\iota}^{-1}\left(t_{j}\right)}{\omega}\right) k\left(\sigma_{\iota}^{-1}\left(t_{j}\right)-\omega s\right) .
$$

THEOREM 3.2. Let $c_{j}$ and $c_{j}^{n}$ be the coefficients defined in (3.3) and (3.6), respectively and let us assume that

$$
\sup _{s \in[a, b]}\left\|\frac{\partial^{2 n}}{\partial t^{2 n}} k\left(\sigma_{2 n}^{-1}(t), \omega s\right)\right\|=\mathcal{C}<\infty
$$

Then, for each $j=1, \ldots, m$, we have

$$
\left|c_{j}(s)-c_{j}^{n}(s)\right| \leq \mathcal{C}\left(1-x_{j}^{2}\right)^{\frac{1}{4}}\left(\frac{s-a}{2}\right) \frac{\pi}{2} \sqrt{\frac{m}{2 n}}\left(\frac{\delta e}{4 n}\right)^{2 n}\left[\left(\frac{m^{2}}{\omega}\right)^{2 n}+1\right] .
$$

Let us remark that the error estimate (3.7) contains the fixed parameter $\omega$, the number of quadrature points $n$, and the degree $m-1$ of the $j$-th Lagrange fundamental polynomial $\ell_{j}$. In practice, since $m^{2} \ll \omega$, the square bracket tends to 1 and the order of convergence is given by $\left(\frac{\delta e}{4 n}\right)^{2 n}$, which quickly tends to zero.

In the next example, in order to make a comparison, we consider again the case examined in Example 3.1, and we compute the coefficients according to formula (3.6).

EXAMPLE 3.3. Let us assume that $k(\omega(t-s))=\frac{\sin (\omega(t-s))}{\omega}, \omega=3000,[a, s] \equiv[-1,1]$, and $m=256$. Table 3.2 shows the approximations obtained by formula (3.6). The results are much more accurate than those given in Table 3.1. 
TABLE 3.2

The approximation of coefficients $c_{j}^{n}(1)$ given in (3.6), for $j=1,5,10$.

\begin{tabular}{c|ccc}
$n$ & $c_{1}^{n}(1)$ & $c_{5}^{n}(1)$ & $c_{10}^{n}(1)$ \\
\hline 8 & $-2.15395840921 \mathrm{e}-08$ & $1.27617252545 \mathrm{e}-07$ & $-5.90164872416 \mathrm{e}-08$ \\
16 & $-2.15388939145 \mathrm{e}-08$ & $1.27624197480 \mathrm{e}-07$ & $-5.90203576187 \mathrm{e}-08$ \\
32 & $-2.15388939145 \mathrm{e}-08$ & $1.27624197480 \mathrm{e}-07$ & $-5.90203576186 \mathrm{e}-08$ \\
64 & $-2.15388939145 \mathrm{e}-08$ & $1.27624197480 \mathrm{e}-07$ & $-5.90203576186 \mathrm{e}-08$
\end{tabular}

3.2. A mixed quadrature scheme. Let us come back to our original integral (3.1). We propose to approximate it by formula (3.2), where the coefficients are computed according to (3.6). Hence, we suggest to write it as

$$
(\mathcal{V} f)(s)=\left(\mathcal{V}_{m}^{n} f\right)(s)+e_{m}^{n}(f, s),
$$

where $\mathcal{V}_{m}^{n}$ is the following dilated product quadrature formula

$$
\left(\mathcal{V}_{m}^{n} f\right)(s)=\sum_{j=1}^{m} c_{j}^{n}(s) f\left(t_{j}\right),
$$

where $c_{j}^{n}$ is defined as in (3.6) and $e_{m}^{n}(f, s)$ is the remainder term.

We remark that such a "mixed quadrature rule" has already been proposed and applied to Fredholm integral equations with nearly singular kernels in [11]. There, the authors give an error estimate in the case $m \equiv n$. Here we provide an estimate for the remainder term in the case $m \neq n$.

THEOREM 3.4. Let $\varphi(t)=\sqrt{(b-t)(t-a)}$. Assume that the kernel function $k$ satisfies the following conditions

$$
\sup _{s \in[a, b]} \int_{a}^{b}|k(t, s)| \log (2+|k(t, s)|) d t<\infty, \quad \sup _{s \in[a, b]} \int_{a}^{b} \frac{|k(t, s)|}{\sqrt{\varphi(t)}} d t<\infty,
$$

and

$$
\max _{t, s \in[a, b]}\left|\frac{\partial^{r} k(t, s)}{\partial t^{r}} \varphi^{r}(t)\right|<+\infty
$$

Then,

$$
\left|(\mathcal{V} f)(s)-\left(\mathcal{V}_{m}^{n} f\right)(s)\right| \leq \mathcal{C} E_{m-1}(f)+\|f\|_{\infty} \sum_{j=1}^{m}\left|c_{j}(s)-c_{j}^{n}(s)\right|,
$$

where $\mathcal{C} \neq \mathcal{C}(n, m, f)$.

REMARK 3.5. Let us underline that, according to Theorem 3.2, for each fixed $m$ and for $n \rightarrow \infty$ the second term of the above estimate goes to zero. Consequently,

$$
\lim _{m \rightarrow \infty}\left(\lim _{n \rightarrow \infty}\left|(\mathcal{V} f)(s)-\left(\mathcal{V}_{m}^{n} f\right)(s)\right|\right)=0
$$

and the order of convergence is given by the error of the best polynomial approximation of $f$. 
4. The numerical method. In this section we describe the numerical method we propose to approximate the solution of equation (1.1). The equation can be rewritten in the operator form

$$
(\mathcal{I}+\mathcal{V}) f=g
$$

where $\mathcal{I}$ is the identity operator and $\mathcal{V}: C^{0}([a, b]) \rightarrow C^{0}([a, b])$ is defined as in (3.1).

Let us consider the following functional equation

$$
\left(\mathcal{I}+\mathcal{V}_{m}^{n}\right) f_{m}^{n}=g
$$

where $f_{m}^{n}$ is unknown and $\mathcal{V}_{m}^{n}$ is defined as in (3.9). By collocating (4.2) at the zeros $s_{i} \in(a, b)$ of the Legendre polynomials, we obtain the linear system of equations

$$
\sum_{j=1}^{m}\left[\delta_{i j}+c_{j}^{n}\left(s_{i}\right)\right] a_{j}=g\left(s_{i}\right), \quad i=1, \ldots, m
$$

where $a_{j}=f_{m}^{n}\left(s_{j}\right)$ are the unknowns and the coefficients $c_{j}^{n}\left(s_{i}\right)$ are defined in (3.6).

Let us note that the previous system of order $m \times m$ can be rewritten in matrix form as

$$
\left(\mathbb{I}_{m}+\mathbb{A}_{m}\right) \mathbf{a}=\mathbf{g}
$$

where $\left[\mathbb{A}_{m}\right]=\left[c_{j}^{n}\left(s_{i}\right)\right]_{i, j=1}^{m}, \mathbf{a}=\left[a_{j}\right]_{j=1}^{m}$, and $\mathbf{g}=\left[g\left(s_{i}\right)\right]_{i=1}^{m}$.

Once solved, its solution $\mathbf{a}^{*}=\left[a_{j}^{*}\right]_{j=1}^{m}$ allows us to compute the Nyström interpolant

$$
f_{m}^{n}(s)=g(s)-\sum_{j=1}^{m} c_{j}^{n}(s) a_{j}^{*}
$$

which approximates the unique solution of equation (1.1).

THEOREM 4.1. Consider the functional equations (4.2). Then, for $m$ sufficiently large and for each fixed $n$, the operators $\left(\mathcal{I}+\mathcal{V}_{m}^{n}\right)$ are invertible and their inverses are uniformly bounded on $C^{0}([a, b])$ with respect to $m$. Moreover, system (4.3) is well conditioned. Furthermore, denote by $\bar{f}$ the unique solution of equation (4.1) and by $\bar{f}_{m}^{n}$ the unique solution of (4.2). Then, if $g \in C^{r}([a, b])$ and the kernel $k$ satisfies the assumptions of Theorem 3.4 , the following error estimate holds true

$$
\left\|\bar{f}-\bar{f}_{m}^{n}\right\|_{\infty}=\mathcal{O}\left(\frac{1}{m^{r}}\right)
$$

where the constants in $\mathcal{O}$ do not depend on $n$ and $m$.

5. Numerical tests. In this section we illustrate with some numerical tests the performance of the method presented in the previous section. We consider three different Volterra integral equations with highly oscillatory kernels. For each test, we solve system (4.3) with $n=20$ and approximate the unique solution of the test equation by means of the Nyström interpolant given by (4.4). Then, we compute the absolute errors. In the first example the exact solution $f^{*}$ is known, thus we compute the errors

$$
\varepsilon_{m}^{n}(s)=\left|f^{*}(s)-f_{m}^{n}(s)\right|,
$$

at different points $s \in[a, b]$. In the other two examples, the exact solution is not available. Then, we consider exact the approximation obtained by the product rule with $m=750$ points and we compute the errors

$$
\varepsilon_{750, m}^{n}(s)=\left|f_{750}^{n}(s)-f_{m}^{n}(s)\right|
$$


ETNA

Kent State University and

Johann Radon Institute (RICAM)

TABLE 5.1

Numerical results for Example 5.1.

\begin{tabular}{c|cccccc}
$\omega$ & $m$ & CPU time & $\varepsilon_{m}^{20}(-0.8)$ & $\varepsilon_{m}^{20}(0.3)$ & $\varepsilon_{m}^{20}(0.7)$ & $\operatorname{cond}\left(\mathbb{I}_{m}+\mathbb{A}_{m}\right)$ \\
\hline $10^{2}$ & 4 & 0.0120 & $7.33 \mathrm{e}-05$ & $7.87 \mathrm{e}-05$ & $3.58 \mathrm{e}-05$ & $1.05 \mathrm{e}+00$ \\
& 8 & 0.0074 & $4.95 \mathrm{e}-09$ & $4.80 \mathrm{e}-09$ & $3.03 \mathrm{e}-09$ & $1.09 \mathrm{e}+00$ \\
& 16 & 0.0178 & M.P. & $6.66 \mathrm{e}-16$ & $4.44 \mathrm{e}-16$ & $1.16 \mathrm{e}+00$ \\
\hline $10^{3}$ & 4 & 0.0336 & $7.09 \mathrm{e}-06$ & $4.67 \mathrm{e}-06$ & $3.22 \mathrm{e}-06$ & $1.01 \mathrm{e}+00$ \\
& 8 & 0.0563 & $3.85 \mathrm{e}-10$ & $2.50 \mathrm{e}-10$ & $1.91 \mathrm{e}-10$ & $1.01 \mathrm{e}+00$ \\
& 16 & 0.1530 & M.P. & $4.44 \mathrm{e}-16$ & $1.78 \mathrm{e}-15$ & $1.01 \mathrm{e}+00$ \\
\hline $10^{4}$ & 4 & 0.2365 & $7.56 \mathrm{e}-07$ & $7.34 \mathrm{e}-08$ & $6.08 \mathrm{e}-07$ & $1.00 \mathrm{e}+00$ \\
& 8 & 0.5445 & $4.21 \mathrm{e}-11$ & $4.19 \mathrm{e}-12$ & $3.39 \mathrm{e}-11$ & $1.00 \mathrm{e}+00$ \\
& 16 & 1.5381 & $6.99 \mathrm{e}-15$ & $1.58 \mathrm{e}-14$ & $3.51 \mathrm{e}-14$ & $1.00 \mathrm{e}+00$
\end{tabular}

Moreover, we also check the infinity norm condition number of the matrix $\mathbb{I}_{m}+\mathbb{A}_{m}$, i.e., $\operatorname{cond}\left(\mathbb{I}_{m}+\mathbb{A}_{m}\right)=\left\|\mathbb{I}_{m}+\mathbb{A}_{m}\right\|_{\infty}\left\|\left(\mathbb{I}_{m}+\mathbb{A}_{m}\right)^{-1}\right\|_{\infty}$. In the tables, the total CPU time of the complete procedure (construction and solution of the linear system+construction and evaluation of the Nyström interpolant) is given. The numerical evidence is that the required CPU time depends on $m$ and $\omega$. If we look at the CPU time for the same value of $m$ in any table, it is clear that the computing time in the case $\omega=10^{3}$ is about 10 times the time for $\omega=10^{2}$.

About the choice of the number of subintervals $N$ arising in the dilation formula (3.6), we found that a good choice is $N=\left\lfloor\frac{\omega(s-a)}{\ell}\right\rfloor$, where $\ell$ is the "wavelength" of the kernel functions. Consequently, the length of the subintervals is $\delta=\frac{\omega(s-a)}{N}$. In practice, the choice of $N$ depends on the length of the integration interval and must take into account the behaviour of the kernel.

In the examples, $\ell=2 \pi$ and, according to $m$ and the collocation point $z_{i}=1, \ldots, m$, if $\omega=10^{2}$ then $N=1, \ldots, 31$, if $\omega=10^{3}$ then $N=1, \ldots, 318$. All the numerical experiments were performed in double precision using Matlab R2019a.

EXAMPLE 5.1. Let us consider the following integral equation

$$
f(s)+\int_{-1}^{s} \cos (\omega(t-s)) f(t) d t=g(s), \quad s \in[-1,1],
$$

with right-hand side $g(s)=e^{s}+\left(e\left(1+\omega^{2}\right)\right)^{-1}\left(e^{s+1}-\cos (\omega(1+s)+\omega \sin (\omega(1+s)))\right.$ and exact solution $f(s)=e^{s}$.

We report in Table 5.1 the approximation errors obtained for different values of the parameter $\omega$. Since the kernel and the right-hand side are analytic functions, the convergence is very fast. The machine precision (M.P.) is attained for $m=16$. Moreover, by the last column of the table, we can observe that the system is extremely well-conditioned.

EXAMPLE 5.2. Let us apply our method to the integral equation

$$
f(s)+\int_{-1}^{s} \frac{J_{1}(\omega(t-s))}{\omega(t-s)} f(t) d t=|\sin (s+3)|^{\frac{11}{2}}, \quad s \in[-1,1],
$$

whose exact solution is not known. Table 5.2 shows that by solving a system of order 256 we get at least 10 correct digits for each fixed $\omega$. Moreover, the condition number of the system does not depend on $m$. 
TABLE 5.2

Numerical results for Example 5.2.

\begin{tabular}{c|cccccc}
$\omega$ & $m$ & CPU time & $\varepsilon_{750, m}^{20}(-0.7)$ & $\varepsilon_{750, m}^{20}(0.4)$ & $\varepsilon_{750, m}^{20}(0.8)$ & cond $\left(\mathbb{I}_{m}+\mathbb{A}_{m}\right)$ \\
\hline $10^{2}$ & 4 & 0.0117 & $2.59 \mathrm{e}-04$ & $9.13 \mathrm{e}-05$ & $2.72 \mathrm{e}-04$ & $1.00 \mathrm{e}+00$ \\
& 8 & 0.0101 & $4.50 \mathrm{e}-06$ & $1.56 \mathrm{e}-05$ & $1.75 \mathrm{e}-06$ & $1.02 \mathrm{e}+00$ \\
& 16 & 0.0229 & $7.79 \mathrm{e}-08$ & $1.11 \mathrm{e}-07$ & $5.80 \mathrm{e}-08$ & $1.04 \mathrm{e}+00$ \\
& 32 & 0.0595 & $6.43 \mathrm{e}-07$ & $2.60 \mathrm{e}-08$ & $3.26 \mathrm{e}-08$ & $1.05 \mathrm{e}+00$ \\
& 64 & 0.1997 & $9.14 \mathrm{e}-08$ & $4.13 \mathrm{e}-08$ & $2.20 \mathrm{e}-09$ & $1.05 \mathrm{e}+00$ \\
& 128 & 0.7873 & $9.58 \mathrm{e}-15$ & $2.80 \mathrm{e}-14$ & $5.59 \mathrm{e}-15$ & $1.04 \mathrm{e}+00$ \\
& 256 & 3.6194 & $8.33 \mathrm{e}-17$ & $3.35 \mathrm{e}-16$ & $8.33 \mathrm{e}-17$ & $1.08 \mathrm{e}+00$ \\
\hline $10^{3}$ & 4 & 0.0459 & $2.71 \mathrm{e}-05$ & $1.09 \mathrm{e}-05$ & $2.48 \mathrm{e}-05$ & $1.00 \mathrm{e}+00$ \\
& 8 & 0.0766 & $5.45 \mathrm{e}-07$ & $1.53 \mathrm{e}-06$ & $6.49 \mathrm{e}-08$ & $1.00 \mathrm{e}+00$ \\
& 16 & 0.1914 & $5.48 \mathrm{e}-09$ & $6.47 \mathrm{e}-10$ & $3.51 \mathrm{e}-09$ & $1.00 \mathrm{e}+00$ \\
& 32 & 0.5583 & $1.76 \mathrm{e}-10$ & $6.42 \mathrm{e}-11$ & $3.13 \mathrm{e}-11$ & $1.01 \mathrm{e}+00$ \\
& 64 & 1.9459 & $1.85 \mathrm{e}-10$ & $1.15 \mathrm{e}-11$ & $3.90 \mathrm{e}-11$ & $1.01 \mathrm{e}+00$ \\
& 128 & 7.8873 & $1.03 \mathrm{e}-11$ & $1.27 \mathrm{e}-12$ & $2.86 \mathrm{e}-11$ & $1.01 \mathrm{e}+00$ \\
& 256 & 34.8418 & $1.52 \mathrm{e}-11$ & $1.97 \mathrm{e}-11$ & $1.49 \mathrm{e}-12$ & $1.01 \mathrm{e}+00$
\end{tabular}

EXAMPLE 5.3. Let us consider the equation

$$
f(s)+\int_{-1}^{s} \frac{\sin (\omega(t-s))}{\omega(t-s)+1} f(t) d t=\left(3 s^{2}+1\right)|s|^{\frac{5}{2}}, \quad s \in[-1,1] .
$$

Here, we have a kernel function which is analytic and a right hand side $g \in C^{2}([-1,1])$. Then, regardless of the parameter $\omega$, the error is of the order $m^{-2}$. This convergence is confirmed by the results of Table 5.3.

TABLE 5.3

Numerical results for Example 5.3.

\begin{tabular}{c|cccccc}
$\omega$ & $m$ & CPU time & $\varepsilon_{750, m}^{20}(-0.5)$ & $\varepsilon_{750, m}^{20}(0.4)$ & $\varepsilon_{750, m}^{20}(0.7)$ & cond $\left(\mathbb{I}_{m}+\mathbb{A}_{m}\right)$ \\
\hline $10^{2}$ & 4 & 0.0125 & $1.63 \mathrm{e}-03$ & $5.09 \mathrm{e}-04$ & $2.32 \mathrm{e}-03$ & $1.00 \mathrm{e}+00$ \\
& 8 & 0.089 & $2.25 \mathrm{e}-07$ & $1.03 \mathrm{e}-07$ & $2.90 \mathrm{e}-06$ & $1.01 \mathrm{e}+00$ \\
& 16 & 0.0222 & $5.70 \mathrm{e}-08$ & $6.25 \mathrm{e}-07$ & $2.08 \mathrm{e}-07$ & $1.03 \mathrm{e}+00$ \\
& 32 & 0.0605 & $7.26 \mathrm{e}-08$ & $1.08 \mathrm{e}-07$ & $8.11 \mathrm{e}-08$ & $1.04 \mathrm{e}+00$ \\
& 64 & 0.2175 & $6.17 \mathrm{e}-08$ & $3.68 \mathrm{e}-08$ & $1.13 \mathrm{e}-08$ & $1.03 \mathrm{e}+00$ \\
& 128 & 0.9046 & $5.77 \mathrm{e}-11$ & $1.08 \mathrm{e}-10$ & $9.98 \mathrm{e}-12$ & $1.03 \mathrm{e}+00$ \\
& 256 & 4.2860 & $7.56 \mathrm{e}-14$ & $3.31 \mathrm{e}-13$ & $6.50 \mathrm{e}-13$ & $1.05 \mathrm{e}+00$ \\
& 512 & 22.1705 & $7.55 \mathrm{e}-15$ & $1.12 \mathrm{e}-13$ & $3.13 \mathrm{e}-14$ & $1.06 \mathrm{e}+00$ \\
\hline $10^{3}$ & 4 & 0.0385 & $1.55 \mathrm{e}-04$ & $5.87 \mathrm{e}-05$ & $2.28 \mathrm{e}-04$ & $1.00 \mathrm{e}+00$ \\
& 8 & 0.0635 & $4.32 \mathrm{e}-08$ & $4.26 \mathrm{e}-08$ & $2.99 \mathrm{e}-07$ & $1.00 \mathrm{e}+00$ \\
& 16 & 0.1726 & $4.33 \mathrm{e}-08$ & $7.38 \mathrm{e}-08$ & $3.61 \mathrm{e}-08$ & $1.00 \mathrm{e}+00$ \\
& 32 & 0.5882 & $9.53 \mathrm{e}-10$ & $4.24 \mathrm{e}-09$ & $2.41 \mathrm{e}-09$ & $1.00 \mathrm{e}+00$ \\
& 64 & 2.0411 & $1.39 \mathrm{e}-10$ & $3.73 \mathrm{e}-11$ & $7.02 \mathrm{e}-12$ & $1.01 \mathrm{e}+00$ \\
& 128 & 8.5121 & $9.83 \mathrm{e}-13$ & $8.85 \mathrm{e}-12$ & $1.79 \mathrm{e}-12$ & $1.01 \mathrm{e}+00$ \\
& 256 & 40.7825 & $1.29 \mathrm{e}-11$ & $2.36 \mathrm{e}-12$ & $6.00 \mathrm{e}-13$ & $1.01 \mathrm{e}+00$ \\
& 512 & 203.9223 & $8.30 \mathrm{e}-12$ & $2.50 \mathrm{e}-13$ & $3.66 \mathrm{e}-13$ & $1.01 \mathrm{e}+00$
\end{tabular}


6. An application. In this section we apply the Nyström-type method described in Section 4 to the direct scattering problem related to $(1.2)$; see $[1,7]$. It consists of computing some initial scattering data, namely, the transmission coefficient $T(\omega)$ and the reflection coefficients from the left $L(\omega)$ and from the right $R(\omega)$. Such a computation involves the solution of certain Volterra integral equations of the form (6.4). In fact, we have

$$
\begin{aligned}
& T(\omega)=\left[1-\frac{1}{2 \mathrm{i} \omega} \int_{\mathbb{R}} q(y) e^{-\mathrm{i} \omega y} f_{\ell}(y) d y\right]^{-1}=\left[1-\frac{1}{2 \mathrm{i} \omega} \int_{\mathbb{R}} q(y) e^{\mathrm{i} \omega y} f_{r}(y) d y\right]^{-1} \\
& R(\omega)=\frac{T(\omega)}{2 \mathrm{i} \omega} \int_{\mathbb{R}} q(y) e^{-\mathrm{i} \omega y} f_{r}(y) d y \\
& L(\omega)=\frac{T(\omega)}{2 \mathrm{i} \omega} \int_{\mathbb{R}} q(y) e^{\mathrm{i} \omega y} f_{\ell}(y) d y
\end{aligned}
$$

where $q$ is the initial potential appearing in (1.2), and $f_{\ell}$ and $f_{r}$ are the solutions of the Volterra integral equations

$$
\begin{cases}f_{\ell}(s)-\frac{1}{\omega} \int_{s}^{\infty} \sin (\omega(t-s)) q(t) f_{\ell}(t) d t=e^{\mathrm{i} \omega s}, & s \in \mathbb{R}, \\ f_{r}(s)-\frac{1}{\omega} \int_{-\infty}^{s} \sin (\omega(s-t)) q(t) f_{r}(t) d t=e^{-\mathrm{i} \omega s}, & s \in \mathbb{R}\end{cases}
$$

with $\omega \in \mathbb{R}^{+} \backslash\{0\}$.

For computing the scattering data $T, L$, and $R$, we need to initially solve equations (6.4), and then approximate the integrals (6.1)-(6.3) by a suitable method. To this end, let us assume that the initial potential $q$ has a bounded support $[a, b]$. This fact implies that (6.4) can be rewritten as

$$
\begin{cases}f_{\ell}(s)-\frac{1}{\omega} \int_{s}^{b} \sin (\omega(t-s)) q(t) f_{\ell}(t) d t=e^{\mathrm{i} \omega s}, & s \leq b, \\ f_{\ell}(s)=e^{\mathrm{i} \omega s}, & s \geq b, \\ f_{r}(s)-\frac{1}{\omega} \int_{a}^{s} \sin (\omega(s-t)) q(t) f_{r}(t) d t=e^{-\mathrm{i} \omega s}, & s \geq a \\ f_{r}(s)=e^{-\mathrm{i} \omega s}, & s \leq a .\end{cases}
$$

Hence, by multiplying both sides of the first integral equation by $q(s) e^{-\mathrm{i} \omega s}$, and the second one by $q(s) e^{\mathrm{i} \omega s}$, we obtain

$$
\begin{cases}F_{\ell}(s)-\frac{1}{\omega} \int_{s}^{b} k_{1}(\omega(t-s)) F_{\ell}(t) d t=q(s), & s \in[a, b], \\ F_{r}(s)-\frac{1}{\omega} \int_{a}^{s} k_{2}(\omega(s-t)) F_{r}(t) d t=q(s), & s \in[a, b],\end{cases}
$$

where $F_{\ell}(s)=q(s) f_{\ell}(s) e^{-\mathrm{i} \omega s}$ and $F_{r}(s)=q(s) f_{r}(s) e^{\mathrm{i} \omega s}$ are the new unknowns, and

$$
k_{1}(\omega(t-s))=\frac{q(s)}{2 \mathrm{i} \omega}\left(e^{2 \mathrm{i} \omega(t-s)}-1\right), \quad k_{2}(\omega(t-s))=\frac{q(s)}{2 \mathrm{i} \omega}\left(e^{2 \mathrm{i} \omega(s-t)}-1\right) .
$$

We point out that multiplicaton of both sides of the equations by $q(s) e^{-\mathrm{i} \omega s}$ (or $q(s) e^{\mathrm{i} \omega s}$ ) has the effect of removing the parameter $\omega$ from the right-hand side and concentrating the 
oscillating terms to the kernel functions only. Indeed, in the original equations (6.4) both the kernel and the right-hand side are highly oscillatory.

At this point, we propose to approximate the solutions of the previous equations by considering the corresponding functional equations (4.2) and solving the related systems (4.3). Once these systems are solved, their solutions $F_{m, \ell}^{n}\left(s_{j}\right)$ (or $F_{m, r}^{n}\left(s_{j}\right)$ ) produce an immediate approximation of the transmission coefficient (6.1) by using the standard Legendre rule (2.14)

$$
T_{m}^{n}(\omega)=\left[1-\frac{1}{2 \mathrm{i} \omega} \sum_{j=1}^{m} \lambda_{j} F_{m, \ell}^{n}\left(s_{j}\right)\right]^{-1}=\left[1-\frac{1}{2 \mathrm{i} \omega} \sum_{j=1}^{m} \lambda_{i} F_{m, r}^{n}\left(s_{j}\right)\right]^{-1} .
$$

Regarding the reflection coefficients (6.2)-(6.3) instead, which can be also rewritten in terms of the functions $F_{\ell}$ and $F_{r}$ as

$$
R(\omega)=\frac{T(\omega)}{2 \mathrm{i} \omega} \int_{a}^{b} e^{-2 \mathrm{i} \omega y} F_{r}(y) d y, \quad L(\omega)=\frac{T(\omega)}{2 \mathrm{i} \omega} \int_{a}^{b} e^{2 \mathrm{i} \omega y} F_{\ell}(y) d y,
$$

we cannot use a standard Legendre rule because of the presence of the oscillating integrand function. Consequently, to approximate them we use the dilation technique applied in Section 4 to the coefficient $c_{k}$. We propose to approximate them as follows

$$
\begin{aligned}
& R_{m}^{n}(\omega)=\frac{T(\omega)}{2 \mathrm{i} \omega} \frac{\delta}{(b-a)} \sum_{\iota=1}^{N} \sum_{j=1}^{m} \lambda_{j} e^{-2 \mathrm{i} \sigma_{\iota}^{-1}\left(s_{j}\right)} F_{m, r}^{n}\left(\frac{\sigma_{\iota}^{-1}\left(s_{j}\right)}{\omega}\right), \\
& L_{m}^{n}(\omega)=\frac{T(\omega)}{2 \mathrm{i} \omega} \frac{\delta}{(b-a)} \sum_{\iota=1}^{N} \sum_{j=1}^{m} \lambda_{j} e^{2 \mathrm{i} \sigma_{\iota}^{-1}\left(s_{j}\right)} F_{m, \ell}^{n}\left(\frac{\sigma_{\iota}^{-1}\left(s_{j}\right)}{\omega}\right),
\end{aligned}
$$

where $\sigma_{\iota}^{-1}(z)=\frac{(z-b)}{(b-a)} \delta+\iota \delta+\omega a$, and $\delta$ is the length of the $N$ subintervals in which we partition the interval $[\omega a, \omega b]$.

In the next two examples we test our method in the case where the initial potential has two different smoothness properties. For both of them we compute the absolute errors

$$
\varepsilon_{m}^{n}(T(\omega))=\left|\left(T^{*}(\omega)-T_{m}^{n}(\omega)\right)\right|, \quad \varepsilon_{m}^{n}(R(\omega))=\left|\left(R^{*}(\omega)-R_{m}^{n}(\omega)\right)\right|,
$$

for different values of $\omega$. Here $T^{*}$ and $R^{*}$ denote the exact transmission and reflection coefficients, respectively. Similar results are obtained for the data $L$.

EXAMPLE 6.1. Let us consider the well-known one-soliton potential [10]

$$
q(x)=-\frac{2}{\cosh ^{2} x},
$$

whose support is $[-18,18]$. The exact forms of the transmission and reflection coefficients are

$$
\begin{aligned}
& T^{*}(\omega)=\frac{\Gamma(2-\mathrm{i} \omega) \Gamma(-1-\mathrm{i} \omega)}{\Gamma(1-\mathrm{i} \omega) \Gamma(-\mathrm{i} \omega)} \\
& R^{*}(\omega)=L^{*}(\omega)=\frac{1}{\pi} \cos \left(\frac{3 \pi}{2}\right) \Gamma(2-\mathrm{i} \omega) \Gamma(-1-\mathrm{i} \omega) \frac{\Gamma(\mathrm{i} \omega)}{\Gamma(-\mathrm{i} \omega)}=0
\end{aligned}
$$

where $\Gamma$ is the Gamma function. Table 6.1 contains the absolute errors. 
TABLE 6.1

Numerical results for Example 6.1.

\begin{tabular}{c|cc|cc|cc}
$m$ & $\omega$ & $\varepsilon_{m}^{n}(T(\omega))$ & $\omega$ & $\varepsilon_{m}^{n}(T(\omega))$ & $\omega$ & $\varepsilon_{m}^{n}(T(\omega))$ \\
\hline 8 & 10 & $1.92 \mathrm{e}-01$ & $10^{2}$ & $1.93 \mathrm{e}-02$ & $10^{3}$ & $1.93 \mathrm{e}-03$ \\
16 & & $1.16 \mathrm{e}-01$ & & $1.16 \mathrm{e}-02$ & & $1.16 \mathrm{e}-03$ \\
32 & & $1.53 \mathrm{e}-02$ & & $1.55 \mathrm{e}-03$ & & $1.55 \mathrm{e}-04$ \\
64 & & $1.13 \mathrm{e}-04$ & & $1.17 \mathrm{e}-05$ & & $1.17 \mathrm{e}-06$ \\
128 & & $2.53 \mathrm{e}-09$ & & $3.14 \mathrm{e}-10$ & & $3.35 \mathrm{e}-11$ \\
256 & & $3.77 \mathrm{e}-15$ & & $2.73 \mathrm{e}-14$ & & $2.60 \mathrm{e}-15$ \\
\hline \hline$m$ & $\omega$ & $\varepsilon_{m}^{n}(R(\omega))$ & $\omega$ & $\varepsilon_{m}^{n}(R(\omega))$ & $\omega$ & $\varepsilon_{m}^{n}(R(\omega))$ \\
\hline 8 & 10 & $9.00 \mathrm{e}-07$ & $10^{2}$ & $7.29 \mathrm{e}-08$ & $10^{3}$ & $2.55 \mathrm{e}-12$ \\
16 & & $7.42 \mathrm{e}-06$ & & $7.18 \mathrm{e}-09$ & & $2.12 \mathrm{e}-11$ \\
32 & & $1.43 \mathrm{e}-05$ & & $9.55 \mathrm{e}-09$ & & $2.58 \mathrm{e}-10$ \\
64 & & $5.36 \mathrm{e}-06$ & & $2.12 \mathrm{e}-09$ & & $3.09 \mathrm{e}-12$ \\
128 & & $1.21 \mathrm{e}-07$ & & $2.66 \mathrm{e}-11$ & & $1.28 \mathrm{e}-13$ \\
256 & & $1.50 \mathrm{e}-11$ & & $3.65 \mathrm{e}-14$ & & $3.07 \mathrm{e}-15$
\end{tabular}

EXAMPLE 6.2. Let us consider the square-well potential

$$
q(x)= \begin{cases}-1, & 0 \leq x \leq 2 \\ 0, & \text { otherwise }\end{cases}
$$

Also in this case we test the performance of our method taking into account the analytical forms of the coefficients

$$
\begin{aligned}
& \frac{1}{T(\omega)}=e^{2 \mathrm{i} \omega}\left\{\cos (2 \beta)-\mathrm{i} \sin (2 \beta)\left(\frac{\beta^{2}+\omega^{2}}{2 \beta \omega}\right)\right\}, \\
& R(\omega)=T(\omega) \mathrm{i} e^{-2 \mathrm{i} \omega} \frac{\sin (2 \beta)}{2 \beta \omega}, \quad L(\omega)=T(\omega) \mathrm{i} e^{2 \mathrm{i} \omega} \frac{\sin (2 \beta)}{2 \beta \omega},
\end{aligned}
$$

where $\beta=\sqrt{1+\omega^{2}}$. Table 6.2 contains the computed errors.

7. Proofs. Proof of Proposition 2.1. Let us set $b_{m, j}:=\left\|P_{m}^{(j)}\right\|_{\infty}$ and prove the statement by induction.

Base case. The statement holds true for $j=1$. Indeed, by (2.8) and by applying (2.9)

$$
b_{m, 1} \leq \sum_{r=0}^{p} c_{m r} b_{m-2 r-1,0}=\sum_{r=0}^{p} c_{m r}=\frac{1}{2} m(m+1) \leq m^{2},
$$

being $b_{m-2 r-1,0}=1$.

Induction hypothesis. Assume that the statement holds true for $j$, i.e., $b_{m, j} \leq m^{2 j}$.

Inductive step. Let us prove that the assertion is true for $j+1$, i.e., $b_{m, j+1} \leq m^{2(j+1)}$. First, let us note that by (2.8) we have

$$
\frac{d^{j+1}}{d x^{j+1}} P_{m}(x)=\sum_{r=0}^{p} c_{m r} \frac{d^{j}}{d x^{j}} P_{m-2 r-1}(x), \quad \text { where } \quad p= \begin{cases}\frac{1}{2}(m-1), & m \text { is odd } \\ \frac{1}{2} m-1, & m \text { is even },\end{cases}
$$

so that, according to the introduced notation, we can write

$$
b_{m, j+1} \leq \sum_{r=0}^{p}\left|c_{m r}\right| b_{m-2 r-1, j}
$$


TABLE 6.2

Numerical results for Example 6.2.

\begin{tabular}{c|cc|cc|cc}
$m$ & $\omega$ & $\varepsilon_{m}^{n}(T(\omega))$ & $\omega$ & $\varepsilon_{m}^{n}(T(\omega))$ & $\omega$ & $\varepsilon_{m}^{n}(T(\omega))$ \\
\hline 8 & 10 & $1.54 \mathrm{e}-04$ & $10^{2}$ & $1.96 \mathrm{e}-08$ & $10^{3}$ & $1.45 \mathrm{e}-10$ \\
16 & & $3.75 \mathrm{e}-07$ & & $5.17 \mathrm{e}-08$ & & $8.80 \mathrm{e}-11$ \\
32 & & $1.39 \mathrm{e}-13$ & & $8.74 \mathrm{e}-08$ & & $5.18 \mathrm{e}-11$ \\
64 & & $6.80 \mathrm{e}-16$ & & $1.79 \mathrm{e}-08$ & & $4.93 \mathrm{e}-11$ \\
128 & & $6.25 \mathrm{e}-16$ & & $4.78 \mathrm{e}-12$ & & $3.26 \mathrm{e}-11$ \\
256 & & $6.94 \mathrm{e}-16$ & & $2.05 \mathrm{e}-15$ & & $1.00 \mathrm{e}-11$ \\
\hline \hline$m$ & $\omega$ & $\varepsilon_{m}^{n}(R(\omega))$ & $\omega$ & $\varepsilon_{m}^{n}(R(\omega))$ & $\omega$ & $\varepsilon_{m}^{n}(R(\omega))$ \\
\hline 8 & 10 & $1.09 \mathrm{e}-05$ & $10^{2}$ & $8.30 \mathrm{e}-09$ & $10^{3}$ & $1.83 \mathrm{e}-09$ \\
16 & & $7.47 \mathrm{e}-06$ & & $1.23 \mathrm{e}-09$ & & $1.25 \mathrm{e}-13$ \\
32 & & $6.12 \mathrm{e}-14$ & & $1.23 \mathrm{e}-09$ & & $1.25 \mathrm{e}-13$ \\
64 & & $1.42 \mathrm{e}-16$ & & $1.17 \mathrm{e}-09$ & & $1.25 \mathrm{e}-13$ \\
128 & & $1.35 \mathrm{e}-16$ & & $9.56 \mathrm{e}-10$ & & $1.25 \mathrm{e}-13$ \\
256 & & $3.75 \mathrm{e}-16$ & & $8.58 \mathrm{e}-18$ & & $1.24 \mathrm{e}-13$
\end{tabular}

By the induction hypothesis, we have

$$
\begin{aligned}
b_{m, j+1} & \leq \sum_{r=0}^{p}[2(m-2 r-1)+1](m-2 r-1)^{2 j} \\
& =\sum_{r=0}^{p} 2(m-2 r-1)^{2 j+1}+\sum_{r=0}^{p}(m-2 r-1)^{2 j}=: S_{1}+S_{2} .
\end{aligned}
$$

Let us estimate $S_{1}$. We can write

$$
\begin{aligned}
& S_{1}=2^{2(j+1)} \sum_{r=0}^{p}\left(\frac{m-1}{2}-r\right)^{2 j+1}=2^{2(j+1)} \begin{cases}\sum_{s=0}^{\frac{m-1}{2}} s^{2 j+1}, & m \text { is odd } \\
\sum_{s=0}^{\frac{m}{2}}\left(s-\frac{1}{2}\right)^{2 j+1}, & m \text { is even },\end{cases} \\
& \leq 2^{2(j+1)} \begin{cases}\left(\frac{m-1}{2}\right)^{2(j+1)}, & m \text { is odd } \\
\frac{m}{2}\left(\frac{m-1}{2}\right)^{2 j+1}, & m \text { is even, }\end{cases}
\end{aligned}
$$

since

$$
\sum_{s=1}^{n} s^{r} \leq n^{r+1}, \quad \sum_{s=1}^{n}\left(s-\frac{1}{2}\right)^{r} \leq n\left(n-\frac{1}{2}\right)^{r} .
$$


Let us now consider $S_{2}$. We have

$$
\begin{aligned}
& S_{2}=2^{2 j} \sum_{r=0}^{p}\left(\frac{m-1}{2}-r\right)^{2 j}=2^{2 j} \begin{cases}\sum_{s=0}^{\frac{m-1}{2}} s^{2 j}, & m \text { is odd }, \\
\sum_{s=0}^{\frac{m}{2}}\left(s-\frac{1}{2}\right)^{2 j}, & m \text { is even, }\end{cases} \\
& \leq 2^{2 j} \begin{cases}\left(\frac{m-1}{2}\right)^{2 j+1}, & m \text { is odd }, \\
\frac{m}{2}\left(\frac{m-1}{2}\right)^{2 j}, & m \text { is even. }\end{cases}
\end{aligned}
$$

By replacing (7.2) and (7.3) into (7.1), we obtain

$$
b_{m, j+1} \leq \begin{cases}(m-1)^{2 j+2}+\frac{1}{2}(m-1)^{2 j+1}, & m \text { is odd }, \\ m(m-1)^{2 j+1}+\frac{m}{2}(m-1)^{2 j}, & m \text { is even. }\end{cases}
$$

Hence, since for each $q \in \mathbb{N}$ the following inequality holds true

$$
(m-1)^{q+1}+\frac{1}{2}(m-1)^{q}=(m-1)^{q}\left(m-\frac{1}{2}\right) \leq m^{q+1},
$$

we have

$$
b_{m, j+1} \leq m^{2(j+1)}
$$

Proof of Proposition 2.2. First, let us note that by (2.12) and (2.10) we can assert that

$$
\ell_{k}(x)=\frac{P_{m}(x)}{P_{m}^{\prime}\left(x_{k}\right)\left(x-x_{k}\right)},
$$

where $x_{k}$ is the $k$-th zero of $p_{m}$. Let us now write the function $\frac{P_{m}(x)}{x-x_{k}}$ as a sum of Legendre orthogonal polynomials, i.e.,

$$
\frac{P_{m}(x)}{x-x_{k}}=\sum_{s=0}^{m-1} c_{m, s}^{k} P_{s}(x)
$$

where, by the orthogonality relation (2.2), we deduce the following form for the coefficients

$$
c_{m, s}^{k}=\frac{2 s+1}{2} \int_{-1}^{1} \frac{P_{m}(x) P_{s}(x)}{x-x_{k}} d x .
$$

By multiplying both sides of (7.5) by $x-x_{k}$ we get

$$
P_{m}(x)=\sum_{s=0}^{m-1} c_{m, s}^{k} P_{s}(x)\left(x-x_{k}\right)=\mathbf{c}_{m}^{k}\left(x-x_{k}\right) \mathbf{P}_{m},
$$


where $\mathbf{c}_{m}^{k}=\left[c_{m, 0}^{k}, c_{m, 1}^{k}, \ldots, c_{m, m-1}^{k}\right]$ and $\mathbf{P}_{m}=\left[P_{0}(x), P_{1}(x), \ldots, P_{m-1}(x)\right]^{T}$.

Then, taking (2.5) into account, we can write

$$
P_{m}(x)=\mathbf{c}_{m}^{k}\left[\left(\mathbb{T}_{m}-x_{k} \mathbb{I}_{m}\right) \mathbf{P}_{m}+\frac{m}{2 m-1} P_{m}(x) \mathbf{e}_{m}\right] .
$$

Hence, we deduce that it must be

(i) $\mathbf{c}_{m}^{k}\left(\mathbb{T}_{m}-x_{k} \mathbb{I}_{m}\right)=\mathbf{0}$;

(ii) $P_{m}(x)=\mathbf{c}_{m}^{k} \frac{m}{2 m-1} P_{m}(x) \mathbf{e}_{n}$, which implies $c_{m, m-1}^{k}=\frac{2 m-1}{m}$.

So, if we consider the linear system $(i)$, by transposition we get

$$
\left(\mathbb{T}_{m}-x_{k} \mathbb{I}_{m}\right)^{T}\left(\mathbf{c}_{m}^{k}\right)^{T}=\mathbf{0}^{T},
$$

and consequently

$$
\left(x_{k} \mathbb{I}_{m}-\mathbb{T}_{m}^{T}\right)\left(\mathbf{c}_{m}^{k}\right)^{T}=\mathbf{0}^{T} .
$$

Thus, since by (ii) we know the coefficient $c_{m, m-1}^{k}$, instead of system (7.6) we can consider the nonsingular left upper $(m-1) \times(m-1)$ block of $\left(x_{k} \mathbb{I}_{m-1}-\mathbb{T}_{m-1}^{T}\right)$, and hence the system

$$
\left(x_{k} \mathbb{I}_{m-1}-\mathbb{T}_{m-1}^{T}\right)\left(\mathbf{c}_{m-1}^{k}\right)^{T}=\alpha_{m-1} c_{m, m-1}^{k} \mathbf{e}_{m-1},
$$

with $\alpha_{m-1}$ as in (2.6). Consequently,

$$
\left(\mathbf{c}_{m-1}^{k}\right)^{T}=\left(x_{k} \mathbb{I}_{m-1}-\mathbb{T}_{m-1}^{T}\right)^{-1} \alpha_{m-1} c_{m, m-1}^{k} \mathbf{e}_{m-1},
$$

namely, the array $\left(\mathbf{c}_{m-1}^{k}\right)^{T}$ is $\alpha_{m-1} c_{m, m-1}^{k}$ times the last column of the inverse matrix $\left(x_{k} \mathbb{I}_{m-1}-\mathbb{T}_{m-1}^{T}\right)^{-1}$.

Let us now focus on the computation of this matrix. To this end, let us denote by

$$
P_{s}(x)=\frac{1}{2^{s}}\left(\begin{array}{c}
2 s \\
s
\end{array}\right) \tilde{P}_{s}(x), \quad \tilde{P}_{s}(x)=\operatorname{det}\left(x \mathbb{I}_{s}-\mathbb{T}_{s}\right),
$$

and by $\mathbb{M}_{s}\left(x_{k}\right)$ the matrix obtained from $x_{k} \mathbb{I}_{m-1}-\mathbb{T}_{m-1}^{T}$ by replacing its $s$-th column by the last column of $I_{m-1}$. Then, by Cramer's rule, for each $s=1,2, \ldots, m-1$, we have

$$
\begin{aligned}
c_{m, s-1}^{k} & =\alpha_{m-1} c_{m, m-1 ; k} \frac{\operatorname{det} \mathbb{M}_{s}\left(x_{k}\right)}{\operatorname{det}\left(x_{k} \mathbb{I}_{m-1}-\mathbb{T}_{m-1}^{T}\right)}=\alpha_{m-1} c_{m, m-1}^{k} \frac{\operatorname{det} \mathbb{M}_{s}\left(x_{k}\right)}{\tilde{P}_{m-1}\left(x_{k}\right)} \\
& =\alpha_{m-1} c_{m, m-1}^{k} \prod_{i=s}^{m-2} \alpha_{i} \frac{\tilde{P}_{s-1}\left(x_{k}\right)}{\tilde{P}_{m-1}\left(x_{k}\right)}
\end{aligned}
$$

and

$$
c_{m, m-2}^{k}=\alpha_{m-1} c_{m, m-1}^{k} \frac{\tilde{P}_{m-2}\left(x_{k}\right)}{\tilde{P}_{m-1}\left(x_{k}\right)} .
$$

Then, taking into account that

$$
\prod_{i=s}^{m-2} \alpha_{i}=\frac{m-1}{2 m-1} \frac{m-2}{2 m-3} \cdots \frac{s}{2 s+1}=\frac{2 s-1}{2 m-1} \frac{\frac{1}{2^{s-1}}\left(\begin{array}{c}
2 s-2 \\
s-1
\end{array}\right)}{\frac{1}{2^{m-1}}\left(\begin{array}{c}
2 m-2 \\
m-1
\end{array}\right)}
$$


we deduce

$$
c_{m, s-1}^{k}=\frac{2 s-1}{m} \frac{P_{s-1}\left(x_{k}\right)}{P_{m-1}\left(x_{k}\right)} .
$$

Consequently, replacing (7.5) and (7.7) in (7.4) yields the following form for the $\ell_{k}$ contribution to the Lagrange interpolation polynomial

$$
\begin{aligned}
\ell_{k}(x) & =\frac{1}{m P_{m}^{\prime}\left(x_{k}\right) P_{m-1}\left(x_{k}\right)} \sum_{s=0}^{m-1}(2 s+1) P_{s}\left(x_{k}\right) P_{s}(x) \\
& =\frac{1}{\sum_{s=0}^{m-1}(2 s+1) P_{s}\left(x_{k}\right)^{2}} \sum_{s=0}^{m-1}(2 s+1) P_{s}\left(x_{k}\right) P_{s}(x) .
\end{aligned}
$$

Then, taking the $j$ th derivative, by applying the Cauchy inequality and Proposition 2.1 we obtain

$$
\begin{aligned}
\left|\ell_{k}^{(j)}(x)\right| & \leq \frac{1}{\sum_{s=0}^{m-1}(2 s+1) P_{s}\left(x_{k}\right)^{2}} \sqrt{\sum_{s=0}^{m-1}(2 s+1)\left[P_{s}\left(x_{k}\right)\right]^{2} \sum_{s=0}^{m-1}(2 s+1)\left[P_{s}^{(j)}(x)\right]^{2}} \\
& =\sqrt{\lambda_{k} \sum_{s=0}^{m-1}(2 s+1)\left[P_{s}^{(j)}(x)\right]^{2}} \\
& \leq m^{2 j} \sqrt{\lambda_{k} \sum_{s=0}^{m-1}(2 s+1)}
\end{aligned}
$$

Finally, taking into account [23, relation (2.3.46)] and [29, relation (15.3.10)],

$$
\lambda_{k} \simeq \frac{\pi}{m} \sqrt{1-x_{k}^{2}},
$$

and

$$
\sum_{s=0}^{m-1}(2 s+1)=\frac{m^{2}}{2}
$$

we deduce the statement, i.e.,

$$
\left|\ell_{k}^{(j)}(x)\right| \leq \sqrt{\frac{\pi}{2}} m^{2 j+\frac{1}{2}}\left(1-x_{k}^{2}\right)^{\frac{1}{4}} .
$$

Proof of Theorem 3.2. By (3.3), (3.6), and (2.14), we have

$$
\left|c_{k}(s)-c_{k}^{n}(s)\right| \leq \frac{\delta I}{(s-a) \omega} \sum_{i=1}^{N}\left|\mathcal{R}_{n}\left(\ell_{k}^{i} k_{s}^{i}, s\right)\right| .
$$


By (2.16), it holds

$$
\left|\mathcal{R}_{n}\left(\ell_{k}^{i} k_{s}^{i}, s\right)\right| \leq\left(\frac{s-a}{2}\right)^{2 n+1} \frac{\pi}{2^{2 n}(2 n) !} \sup _{t \in[a, s]}\left|\frac{\partial^{2 n}}{\partial t^{2 n}}\left[\ell_{j}\left(\frac{\sigma_{i}^{-1}(t)}{\omega}\right) k\left(\sigma_{i}^{-1}(t)-\omega s\right)\right]\right| .
$$

Moreover, by the Leibniz rule and Proposition 2.2, we have

$$
\begin{aligned}
& \left|\frac{\partial^{2 n}}{\partial t^{2 n}}\left[\ell_{j}\left(\frac{\sigma_{i}^{-1}(t)}{\omega}\right) k\left(\sigma_{i}^{-1}(t)-\omega s\right)\right]\right| \\
& \leq \sum_{j=0}^{2 n}\left(\begin{array}{c}
2 n \\
j
\end{array}\right)\left[\ell_{j}\left(\frac{\sigma_{i}^{-1}(t)}{\omega}\right)\right]^{(2 n-i)}\left|\frac{\partial^{i}}{\partial t^{i}} k\left(\sigma_{i}^{-1}(t)-\omega s\right)\right| \\
& \leq \sup _{t, s \in[a, b]}\left|\frac{\partial^{2 n}}{\partial t^{2 n}} k\left(\sigma_{2 n}^{-1}(t)-\omega s\right)\right| \sqrt{\frac{m \pi}{2}}\left(1-x_{j}^{2}\right)^{\frac{1}{4}} \sum_{j=0}^{2 n}\left(\begin{array}{c}
2 n \\
j
\end{array}\right)\left(\frac{\delta m^{2}}{(s-a) \omega}\right)^{2 n-i}\left(\frac{\delta}{s-a}\right)^{i} \\
& =\mathcal{C} \sqrt{\frac{m \pi}{2}}\left(1-x_{j}^{2}\right)^{\frac{1}{4}}\left(\frac{\delta}{s-a}\right)^{2 n}\left(\frac{m^{2}}{\omega}+1\right)^{2 n}
\end{aligned}
$$

where $\mathcal{C}=\sup _{s \in[a, b]}\left\|\frac{\partial^{2 n}}{\partial t^{2 n}} k\left(\sigma_{i}^{-1}(t)-\omega s\right)\right\|$. Therefore, by (7.8) and (2.15), we have

$$
\begin{aligned}
\left|\mathcal{R}_{n}\left(\ell_{k}^{i} k_{y}^{i}, y\right)\right| & \leq \mathcal{C}\left(1-x_{j}^{2}\right)^{\frac{1}{4}}\left(\frac{s-a}{2}\right) \sqrt{\frac{m \pi}{2}} \frac{\pi}{(2 n) !}\left[\frac{\delta}{2^{2 n}}\left(\frac{m^{2}}{\omega}+1\right)\right]^{2 n} \\
& \leq \mathcal{C}\left(1-x_{j}^{2}\right)^{\frac{1}{4}}\left(\frac{s-a}{2}\right) \frac{\pi}{2} \sqrt{\frac{m}{2 n}}\left(\frac{\delta e}{4 n}\right)^{2 n}\left[\left(\frac{m^{2}}{\omega}\right)^{2 n}+1\right]
\end{aligned}
$$

since $(a+b)^{p} \leq 2^{p}\left(a^{p}+b^{p}\right)$ for each $a, b>0$.

Proof of Theorem 3.4. By (3.8),

$$
\left|e_{m}^{n}(f, s)\right|=\left|(\mathcal{V} f)(s)-\left(\mathcal{V}_{m}^{n} f\right)(s)\right| \leq\left|(\mathcal{V} f)(s)-\left(\mathcal{V}_{m} f\right)(s)\right|+\left|\left(\mathcal{V}_{m} f\right)(s)-\left(\mathcal{V}_{m}^{n} f\right)(s)\right|,
$$

where $\mathcal{V}_{m}$ is defined in (3.2). By applying Theorem 5.1.12 in [23] and taking (3.9) into account we can write

$$
\begin{aligned}
\left|e_{m}^{n}(f, s)\right| & \leq \mathcal{C} E_{m-1}(f, s)+\sum_{k=1}^{m}\left|c_{k}(s)-c_{k}^{n}(s)\right|\left|f\left(t_{k}\right)\right| \\
& \leq \mathcal{C} E_{m-1}(f, y)+\|f\|_{\infty} \sum_{k=1}^{m}\left|c_{k}(s)-c_{k}^{n}(s)\right| .
\end{aligned}
$$

Proof of Theorem 4.1. First, let us note that the operator $\mathcal{V}: C^{0}([a, b]) \rightarrow C^{0}([a, b])$ is compact and the sequence of operators $\left\{\mathcal{V}_{m}^{n}\right\}_{m}$ is collectively compact; see, for instance, [2, 3]. Moreover, by virtue of Theorem 3.4, $\left\|\left(\mathcal{V}-\mathcal{V}_{m}^{n}\right) f\right\|_{\infty} \rightarrow 0$ for $m \rightarrow \infty$ and for each fixed $n$. Following [2], we deduce that, for each fixed $n$, the operators $\left(I+\mathcal{V}_{m}^{n}\right)^{-1}$ exist and are uniformly bounded with respect to $m$. Regarding the well-conditioning of the matrix $\mathbb{I}_{m}+\mathbb{A}_{m}$, by using the same arguments as [4, p. 113], we can prove that

$$
\operatorname{cond}\left(\mathbb{I}_{m}+\mathbb{A}_{m}\right) \leq \operatorname{cond}\left(I+\mathcal{V}_{m}^{n}\right)=\left\|I+\mathcal{V}_{m}^{n}\right\|\left\|\left(I+\mathcal{V}_{m}^{n}\right)^{-1}\right\|
$$


Now, denote by $\bar{f}$ the unique solution of equation (4.1) and by $\bar{f}_{m}^{n}$ the unique solution of (4.2). From (4.1) and (4.2) we deduce that

$$
\left\|\bar{f}-\bar{f}_{m}^{n}\right\|_{\infty} \leq\left\|\left(\mathcal{V}-\mathcal{V}_{m}^{n}\right) f\right\|_{\infty},
$$

so that by applying Theorem 3.4 and the assumptions we obtain the thesis.

Acknowledgments. The authors are grateful to the reviewers for carefully reading the manuscript and suggesting some improvements.

\section{REFERENCES}

[1] T. AKtosun, M. Klaus, AND C. VAn DER MeE, Factorization of scattering matrices due to partitioning of potentials in one-dimensional Schrödinger-type equations, J. Math. Phys., 37 (1996), pp. 5897-5915.

[2] P. M. ANSELone, Uniform approximation theory for integral equations with discontinuous kernels, SIAM J. Numer. Anal., 4 (1967), pp. 245-253.

[3] Collectively compact approximations of integral operators with discontinuous kernels, J. Math. Anal. Appl., 22 (1968), pp. 582-590.

[4] K. E. AtKInSON, The Numerical Solution of Integral Equations of the Second Kind, Cambridge University Press, Cambridge, 1997.

[5] P. BARATELla AND A. P. ORSI, A new approach to the numerical solution of weakly singular Volterra integral equations, J. Comput. Appl. Math., 163 (2004), pp. 401-418.

[6] H. BRUNNER, Y. MA, AND Y. XU, The oscillation of solutions of Volterra integral and integro-differential equations with highly oscillatory kernels, J. Integral Equations Appl., 27 (2015), pp. 455-487.

[7] K. ChADAN AND P. SABATIER, Inverse Problems in Quantum Scattering Theory, 2nd ed., Springer, New York, 1989.

[8] D. L. Colton And R. KRess, Integral Equation Methods in Scattering Theory, Wiley, New York, 1983.

[9] M. C. DE BONIS AND P. PASTORE, A quadrature formula for integrals of highly oscillatory functions, Rend. Circ. Mat. Palermo (2) Suppl., 82 (2010), pp. 279-303.

[10] P. G. DRaZin And R. S. Johnson, Solitons: An Introduction, Cambridge University Press, Cambridge, 1989.

[11] L. Fermo, M. G. Russo, AND G. Serafini, Numerical treatment of the generalized Love integral equation, Numer. Algorithms, 86 (2021), pp. 1769-1789.

[12] L. N. G. FILON, On a quadrature formula for trigonometric integrals, Proc. R. Soc. Edinb., 49 (1928), pp. 38-47.

[13] E. A. FLINN, A modification of Filon's method of numerical integration, J. ACM, 7 (1960), pp. 181-184.

[14] N. K. Govil AND R. N. MoHAPATRA, Markov and Bernstein type inequalities for polynomials, J. Inequal. Appl., 3 (1999), pp. 349-387.

[15] D. HuYBRECHS AND S. VANDEWALLE, On the evaluation of highly oscillatory integrals by analytic continuation, SIAM J. Numer. Anal., 44 (2006), pp. 1026-1048.

[16] A. ISERLES AND S. P. NøRSETT, On quadrature methods for highly oscillatory integrals and their implementation, BIT, 44 (2004), pp. 755-772.

[17] - Efficient quadrature of highly oscillatory integrals using derivatives, Proc. R. Soc. London Ser. A-Math. Phys. Eng. Sci., 461 (2005), pp. 1383-1399.

[18] S. KABANiKhIn AND A. LoRENZI, Identification Problems of Wave Phenomena, VSP, Utrecht, 1999.

[19] D. KorTEWEG AND G. DE VRIES, On the change of form of long waves advancing in a rectangular channel and on a new type of long stationary waves, Philos. Mag., 39 (1895), pp. 422-443.

[20] D. LeVIN, Fast integration of rapidly oscillatory functions, J. Comput. Appl. Math., 67 (1996), pp. 95-101.

[21] _ Analysis of a collocation method for integrating rapidly oscillatory functions, J. Comput. Appl. Math., 78 (1997), pp. 131-138.

[22] Y. Ma AND Y. XU, Computing highly oscillatory integrals, Math. Comp., 87 (2018), pp. 309-345.

[23] G. Mastroianni And G. V. Milovanović, Interpolation Processes, Springer, Berlin, 2008.

[24] D. OCCORSIO AND G. SERAFINI, Cubature formulae for nearly singular and highly oscillating integrals, Calcolo, 55 (2018), Paper No. 4, 33 pages.

[25] S. OLVER, Moment-free numerical approximation of highly oscillatory functions IMA J. Numer. Anal., 26 (2006), pp. 213-227.

[26] _ Moment-free numerical approximation of highly oscillatory integrals with stationary points, Eur. J. Appl. Math., 18 (2007), pp. 435-447.

[27] P. PASTORE, The numerical treatment of Love's integral equation having very small parameter, J. Comput. Appl. Math., 236 (2011), pp. 1267-1281. 
[28] I. N. SNEdDon, Special Functions of Mathematical Physics and Chemistry, Oliver and Boyd, Edinburg and London, 1956.

[29] G. SzEgŐ, Orthogonal Polynomials, 4th ed., American Mathematical Society, Providence, 1975.

[30] A. F. Timan, Theory of Approximation of Functions of a Real variable, Dover, New York, 1994.

[31] S. XIANG AND H. BRUNNER, Efficient methods for Volterra integral equations with highly oscillatory Bessel kernels, BIT, 53 (2013), pp. 241-263.

[32] S. Xiang, Y. J. Cho, H. WANG, And H. Brunner, Clenshaw-Curtis-Filon-type methods for highly oscillatory Bessel transforms and applications, IMA J. Numer. Anal., 31 (2011), pp. 1281-1314. 